\title{
Nível de satisfação dos usuários e verificação do conhecimento dos farmacêuticos em farmácias públicas do Espírito Santo, Brasil
}

\author{
Satisfaction levels of users and verification of the knowledge of \\ pharmacists in public pharmacies in the State of Espírito Santo, Brazil
}

Raphael Laiber Bonadiman ${ }^{1}$ Adriélly Fiorese Santanna ${ }^{1}$ Girlandia Alexandre Brasil ${ }^{1}$ Ewelyne Miranda de Lima ${ }^{1}$ Dominik Lenz ${ }^{1}$ Denise Coutinho Endringer ${ }^{1}$ Tadeu Uggere Andrade ${ }^{1}$

${ }^{1}$ Complexo Biopráticas, Universidade Vila Velha. R. Mercúrio, Residencial Coqueiral. 29102-623 Vila Velha ES Brasil. tadeu.andrade@uvv.br

\begin{abstract}
The scope of this study was to determine the satisfaction levels of users of public pharmacies and verify the knowledge of pharmacists in relation to dispensing of medicines. This is a descriptive study, conducted in municipalities in the State of Espirito Santo, in the period from May to August 2013. Data were collected using a structured questionnaire and SPSS20 software was used for statistical analysis. The Student $t$ or ANOVA test was used for data with normal distribution, while the Mann-Whitney or Kruskal-Wallis test was applied for data without normal distribution. The Spearman correlation was used to evaluate patient satisfaction with the service time and the waiting time. The significance level for the tests was 5\%. The main results obtained were the high level of dissatisfaction among users and the correlation with the waiting time to be attended. Apart from this, the knowledge of pharmacists in relation to the medication dispensed was classified as satisfactory/regular. The conclusion drawn is that actions are necessary in the management of pharmaceutical service to address the expectations of users in the dispensation of drugs.
\end{abstract}

Key words Community pharmacy services, $\mathrm{Pa}$ tient satisfaction, Health services, Education in drug dispensation
Resumo O objetivo do presente estudo foi determinar o nível de satisfação dos usuários de farmácias públicas e verificar o conhecimento dos farmacêuticos com relação à dispensação de medicamentos. Trata-se de um estudo descritivo, realizado em municípios de uma mesma região, no interior do Estado do Espírito Santo, no período de maio a agosto de 2013. Os dados foram coletados por meio de questionário estruturado. Para a análise estatística, foi utilizado o Software SPSS20. Para os dados que apresentaram distribuição normal foram aplicados o teste $t$ student ou ANOVA, enquanto que para os demais foram aplicados os testes Mann-Whitney ou Kruskal -Wallis. A correlação de Spearman foi utilizada para avaliar a satisfação do paciente em relação ao tempo de atendimento e ao tempo de espera. O nivel de significância adotado para os testes foi de 5\%. Os principais resultados obtidos foram o alto nível de insatisfação por parte dos usuários e a correlação com o tempo de espera até o atendimento. Além disso, o conhecimento dos farmacêuticos em relação à dispensação foi classificado como satisfatório/regular. Conclui-se que são necessárias medidas na gestão de serviços farmacêuticos para atender as expectativas dos usuários na dispensação de medicamentos.

Palavras-chave Serviços comunitários de farmácia, Satisfação do paciente, Serviços de saúde, Educação em farmácia 


\section{Introdução}

A avaliação dos serviços de saúde, em geral, é uma prática sistemática muito recente no cenário mundial. As tentativas de sistematizar a avaliação dos serviços de saúde eram concentradas às práticas médicas e ao controle do exercício profissional. Deste modo, como não eram tidos como essenciais ao funcionamento dos serviços, os estudos de avaliação se desenvolveram vagarosamente e de forma fracionada ${ }^{1}$. No cenário atual, pesquisa sobre satisfação dos usuários tem sido considerada um importante indicador dos serviços de saúde, por meio do qual é possível acessar a qualidade e a aplicabilidade desses serviços².

Nesse contexto, os serviços de saúde podem ser avaliados de várias maneiras, tanto pela estrutura física e mão-de-obra humana e material, quanto às atividades realizadas com o intuito de fornecer melhoria terapêutica ao paciente e relações interpessoais. Além disso, o resultado obtido pelo paciente também deve ser levado em consideração ${ }^{3}$.

A verificação da satisfação dos usuários tem sido muito utilizada em países desenvolvidos como um instrumento multidimensional de qualidade de serviços farmacêuticos e de saúde ${ }^{4,5}$. No entanto, esse tipo de estudo no Brasil ainda é uma ferramenta pouco explorada.

Os serviços farmacêuticos passaram por uma grande transformação, mudando seu foco do produto para os cuidados com os pacientes ${ }^{6}$, por isso é necessário que estes, tenham um conhecimento satisfatório acerca dos medicamentos, da farmacoterapia, das boas práticas em farmácia, agindo sempre pautado nos princípios éticos e contribuindo assim para o simples ato da entre$\mathrm{ga}^{7}$.

Na prática dos serviços farmacêuticos, Lucchetta e Mastroianni ${ }^{8}$ reportaram um contexto de desmotivação e de excesso de atividades administrativas e, ainda, nível de conhecimento regular sobre a legislação profissional e sanitária, sinalizando um grave problema na execução desses serviços, podendo comprometer a sua qualidade e dessa forma reduzir o nível de satisfação com os serviços prestados. Adicionalmente, Silva e Vieira ${ }^{9}$ alertam que a deficiência do conhecimento pode gerar distorções do verdadeiro papel do profissional.

Os serviços farmacêuticos prestados podem ampliar o conhecimento do paciente acerca de sua condição de saúde, melhorar sua adesão ao tratamento e sua avaliação pode ser utilizada para verificar o impacto e a qualidade desses serviços ${ }^{6,10,11}$. De acordo com Andrade et al. ${ }^{12}$, os serviços farmacêuticos podem desempenhar um papel determinante no nível de satisfação dos usuários.

Diante do exposto, a necessidade de estudos, as poucas evidências científicas que relatam a satisfação dos usuários e a realidade do profissional farmacêutico nas farmácias públicas, direcionou a pesquisa para determinar o nível de satisfação dos usuários das farmácias públicas municipais, de dispensação de medicamentos básicos, e verificar o conhecimento dos farmacêuticos com relação à dispensação desses medicamentos em cidades no interior do Espírito Santo.

\section{Métodos}

\section{Tipo de estudo e amostragem}

Estudo descritivo, com abordagem quantitativa, onde os dados foram coletados por meio de questionários estruturados. O presente estudo foi realizado em municípios de uma mesma região de saúde do Estado do Espírito Santo, no período de maio a agosto de 2013.

O Plano Diretor de Regionalização da Saúde do Espírito Santo ${ }^{13}$, que divide o estado em quatro regiões (norte, central, metropolitana e sul), foi utilizado para seleção da amostra. Escolheu-se a região sul, composta por 623.396 mil habitantes distribuídos em 26 municípios. Foram selecionados 8 municípios, com probabilidade proporcional ao tamanho, correspondendo a $351.196 \mathrm{mil}$ habitantes.

O tamanho da amostra, para se chegar ao nível de satisfação dos usuários, foi determinado com o uso do software livre EpiInfo ${ }^{\circ} \mathrm{V}$ 7.0, com base na média mensal de atendimentos de cada farmácia. Ao todo, 15.200 atendimentos mensais são realizados nos oito municípios, conforme dados fornecidos pelas secretarias de saúde. Foi considerado nível de confiança de $95 \%$, resultando em 247 indivíduos, e selecionados 294 para reposição de perdas. A amostra calculada foi estratificada por cada farmácia, de acordo com a sua população adstrita.

Nas 8 unidades de farmácia escolhidas haviam um total de 13 farmacêuticos convidados a participar da entrevista. Destes, 2 se recusaram, e a amostra final foi de 11 farmacêuticos. 


\section{Descrição do serviço}

A Assistência Farmacêutica é um dos eixos estratégicos da Política Nacional de Medicamentos $^{14}$ e foi normatizada pela Política Nacional de Assistência Farmacêutica ${ }^{15}$. Os medicamentos disponibilizados pelo SUS são divididos em três categorias: básico, especializado e estratégico ${ }^{16}$.

A dispensação gratuita de medicamentos do componente básico é de responsabilidade dos municípios e podem ocorrer em farmácias públicas municipais (farmácias básicas) e/ou em farmácias de unidades básicas de saúde, ambas as partes integrantes da atenção primária à saúde do SUS. O profissional farmacêutico deve possuir conhecimento satisfatório acerca dos medicamentos, da farmacoterapia, das boas práticas em farmácia e agir sempre pautado nos princípios éticos e normativos que regulamentam sua profissão ${ }^{7}$.

Os medicamentos do componente básico constam da relação nacional de medicamentos e/ou fazem parte do perfil nosológico de cada município e, neste caso, deve também constar de relação municipal de medicamentos. Para ter acesso a um medicamento da atenção básica, o paciente precisa ser atendido em uma unidade básica de saúde ou em hospital do SUS e ter uma prescrição médica.

\section{Seleção da amostra}

Para a seleção da amostra, foram adotados procedimentos intencionais por conveniência, para pacientes ou acompanhantes que buscaram o atendimento na farmácia básica de seu respectivo município. Cada farmácia foi visitada em dias aleatórios para a realização da pesquisa, porém, sempre no mesmo período do mês. Adicionalmente, os pacientes foram caracterizados com o município de residência, idade, sexo, escolaridade e renda familiar; além de informações sobre os motivos que levaram à procura da farmácia, tempo de espera e tempo de duração do atendimento.

\section{Critérios de inclusão e exclusão}

Foram incluídos pacientes ou acompanhantes que já frequentavam as farmácias básicas dos respectivos municípios, com idade acima de 18 anos e os farmacêuticos responsáveis técnicos pelo estabelecimento.

Foram excluídos pacientes que não possuíram condições de responder o questionário.

\section{Instrumento de determinação da satisfação}

Para determinar a satisfação dos usuários, foi aplicado um questionário estruturado, "Questionário de Satisfação com os serviços da farmácia”, elaborado e implantado, primeiramente, por $\mathrm{Ku}$ cukarslan e Schommer ${ }^{17}$ e adaptado, traduzido e validado para a língua portuguesa por Correr et al. ${ }^{6}$

O questionário foi dividido em dois blocos de perguntas, o primeiro com oito perguntas relacionadas à dispensação e aos aspectos estruturais da farmácia, como: a aparência, a qualidade do atendimento, a disponibilidade e qualidade das respostas do farmacêutico, sua relação com o paciente e a cortesia e o respeito de todos os funcionários.

O segundo bloco referia-se aos serviços prestados pelos farmacêuticos, com doze itens relacionados à atuação deste, como: orientação e responsabilidade com o paciente, o interesse, resolução de problemas, qualidade das orientações, privacidade no atendimento e o tempo dispensado ao paciente ${ }^{6}$.

Foi utilizada uma escala de intensidade de cinco pontos do tipo Likert para cada pergunta (num total de 20), onde o menor número representa à opção "péssimo", e o maior a opção "muito bom". Os usuários foram classificados como satisfeitos quando a pontuação foi igual ou maior que 4 , e insatisfeitos quando menor que 4 .

\section{Instrumento de verificação do nível de conhecimento do profissional farmacêutico}

Para a coleta de dados foi utilizado um questionário estruturado e validado por Reis ${ }^{18}$, com modificações, para verificar a contribuição dos farmacêuticos com dispensação e realização da Atenção Farmacêutica. As perguntas sobre aspectos comerciais foram excluídas para adaptação do questionário para rede pública.

Dividiu-se 35 questões em três seções: dados demográficos, conhecimento e conduta. $\mathrm{Na}$ primeira seção, as variáveis foram: idade, sexo, tipo de instituição de formação do profissional, experiência em dispensação, carga horária laborativa, remuneração, benefícios salariais, habilitação, tempo de ocupação no cargo, vínculos de trabalho e a participação dos farmacêuticos em atividades de educação continuada.

O conhecimento sobre dispensação foi avaliado por meio de cinco perguntas objetivas, com apenas uma opção correta, sobre os temas: medicamentos sujeitos a controle especial, dispensa- 
ção de antimicrobianos, orientação do farmacêutico sobre a utilização dos medicamentos, descarte de medicamentos e o conceito de Atenção Farmacêutica. Assim, de acordo com o número de respostas corretas, o conhecimento foi classificado como insatisfatório (0-2 corretas), regular (3-corretas) e satisfatório (4-5 corretas). Além disso, os profissionais também foram questionados sobre a realização da atenção farmacêutica, registro de pacientes, orientações, intervenções farmacêuticas e estrutura física da farmácia para prática profissional.

A terceira seção continha questões objetivas relacionadas com a conduta farmacêutica: frequência e tempo gasto em atividades burocráticas, orientação, limpeza de prateleiras, reposição e aquisição de produtos, verificação da validade, condutas durante a dispensação de medicamentos. Os participantes também responderam a uma questão sobre como eles se avaliavam enquanto agentes de saúde comprometidos com o sucesso da farmacoterapia e qualidade de vida do paciente.

\section{Análise estatística e aspectos éticos}

Todos os dados coletados foram compilados em planilha elaborada no Microsoft Excel ${ }^{\hat{a}}$ versão Office 2007 e analisados pelo programa estatístico StatisticalPackage Social Science versão 20 (SPSS 20). Foram realizadas as frequências relativas simples para as variáveis qualitativas dentro de cada grupo e comparação entre os grupos (frequências relativas cruzadas). $\mathrm{O}$ teste de Kolmogorov-Sminorv foi utilizado para testar a normalidade das variáveis contínuas. Nas variáveis com apenas duas condições (sexo e dispensação de medicamentos) foi utilizado o teste $\mathrm{t}$ (paramétrico) e teste Mann-Whitney U (não paramétrico). Nas variáveis com mais de duas condições (escolaridade, renda, idade, tempo de espera e tempo de atendimento) foi utilizado o teste ANOVA de 1 via (paramétrico) e teste Kruskal-Wallis (não paramétrico). A correlação de Spearman foi utilizada para avaliar a satisfação (média \pm E.P.M.) do paciente em relação ao tempo de atendimento e tempo de espera. O nível de significância foi de $5 \%$.

O trabalho foi aprovado pelo Comitê de Ética e Pesquisa em Seres Humanos da Universidade Vila Velha (UVV).

\section{Resultados}

\section{Descrição da amostra}

As características sociodemográficas e outras variáveis dos pacientes atendidos nas farmácias estão na Tabela 1. Do total dos 294 pacientes que participaram do estudo, a maioria eram mulheres, com idade entre 35 e 49 anos, recebiam de 1 a 2 salários mínimos e possuíam ensino médio completo. A procura da maioria dos pacientes pelas farmácias se faz sempre e a origem da prescrição é do SUS.

\section{Dispensação dos medicamentos}

Dos pacientes, 264 conseguiram obter os medicamentos no momento da dispensação, representando a maioria da amostra. $\mathrm{O}$ fato de não haver o medicamento foi o principal motivo da não dispensação. O outro motivo da não dispensação foi a documentação incompleta por parte dos usuários.

\section{Satisfação}

Na Tabela 2 estão apresentados os dados para cada pergunta do questionário de acordo com a escala de Likert. A média geral foi de 2,96 $\pm 0,91$. A média da pontuação em todas as questões foi inferior a 3,3, sendo as piores médias obtidas nas questões 1,17 e 20 . Deste modo, os usuários foram classificados como insatisfeitos, pois a média geral apresentou-se menor que 4 .

\section{Tempo de espera e duração do atendimento}

O tempo médio de espera pelo atendimento foi de 12,7 $\pm 8,2$, em minutos, e variou de 5,6 \pm 2,3 min. a 16,1 \pm min (Tabela 3). O tempo médio de duração do atendimento pelo farmacêutico, em minutos, foi de $4,4 \pm 2,3$, e variou de $4,1 \pm$ 2,4 min. a 5,5 $\pm 3,6 \min$ (Tabela 3 ).

\section{Relação entre satisfação e outras variáveis}

Na tabela 3 estão descritas as análises de variáveis sociodemográficas, tempo de espera pelo atendimento, tempo de atendimento pelo farmacêutico e dispensação de medicamentos em relação à satisfação dos usuários. Na variável tempo de espera pelo atendimento, não houve usuários satisfeitos quando o tempo superou os 20 minutos. Também houve correlação significativa com 
Tabela 1. Distribuição percentual das variáveis selecionadas dos pacientes atendidos nas farmácias básicas do sul do Espírito Santo.

\begin{tabular}{|c|c|c|c|}
\hline Variáveis & Categorias & Frequências (n) & Porcentagens (\%) \\
\hline \multirow[t]{2}{*}{ Gênero } & Feminino & 202 & $68,7 \%$ \\
\hline & Masculino & 92 & $31,3 \%$ \\
\hline \multirow[t]{5}{*}{ Idade } & 18 a 34 anos & 61 & $20,7 \%$ \\
\hline & 35 a 49 anos & 100 & $34,0 \%$ \\
\hline & 50 a 60 anos & 88 & $29,9 \%$ \\
\hline & 61 a 70 anos & 32 & $10,9 \%$ \\
\hline & 71 a 80 anos & 13 & $4,4 \%$ \\
\hline \multirow{5}{*}{ Renda } & $<1$ salário mínimo & 60 & $20,4 \%$ \\
\hline & 1 a 2 salários mínimos & 198 & $67,3 \%$ \\
\hline & 3 a 5 salários mínimos & 34 & $11,6 \%$ \\
\hline & 6 a 10 salários mínimos & 1 & $0,3 \%$ \\
\hline & > 10 salários mínimos & 1 & $0,3 \%$ \\
\hline \multirow[t]{6}{*}{ Escolaridade } & Analfabeto & 15 & $5,1 \%$ \\
\hline & Ensino fundamental completo & 62 & $21,1 \%$ \\
\hline & Ensino fundamental incompleto & 63 & $21,4 \%$ \\
\hline & Ensino médio completo & 100 & $34,0 \%$ \\
\hline & Ensino médio incompleto & 38 & $12,9 \%$ \\
\hline & Ensino superior completo & 16 & $5,4 \%$ \\
\hline \multirow[t]{3}{*}{ Procura pela farmácia } & Primeira vez & 12 & $4,1 \%$ \\
\hline & Às vezes & 138 & $46,9 \%$ \\
\hline & Sempre & 144 & $49,0 \%$ \\
\hline \multirow[t]{8}{*}{ Município } & Anchieta & 20 & $6,8 \%$ \\
\hline & Cachoeiro do Itapemirim & 177 & $60,2 \%$ \\
\hline & Iconha & 11 & $3,7 \%$ \\
\hline & Itapemirim & 25 & $8,5 \%$ \\
\hline & Marataízes & 28 & $9,5 \%$ \\
\hline & Piúma & 14 & $4,8 \%$ \\
\hline & Presidente Kennedy & 9 & $3,1 \%$ \\
\hline & Rio Novo Sul & 10 & $3,4 \%$ \\
\hline \multirow[t]{2}{*}{ Origem da prescrição } & SUS & 293 & $99,7 \%$ \\
\hline & Particular & 1 & $0,3 \%$ \\
\hline \multirow[t]{3}{*}{ Caso Negativo } & Não há medicamento disponível & 29 & $9,9 \%$ \\
\hline & Documentação incompleta & 1 & $0,3 \%$ \\
\hline & Não houve caso negativo & 264 & $89,8 \%$ \\
\hline
\end{tabular}

a renda, a escolaridade, a faixa etária e o fato de o medicamento ter ou não sido dispensado.

\section{Correlação entre o tempo de espera e satisfação}

Houve uma correlação negativa entre o tempo de espera pelo atendimento e o nível de satisfação, ou seja, quanto maior o tempo de espera pelo atendimento menor a satisfação dos usuários $(r=-0,518 ; p<0,001)$. Em relação ao tempo de duração do atendimento pelo farmacêutico e a satisfação, não houve correlação significativa $(r$ $=0,094 ; p=0,144)$.

\section{Dados gerais dos farmacêuticos}

A maioria dos farmacêuticos encontrava-se na faixa etária de 20 a 30 anos $(81,8 \%)$, pertence ao sexo feminino $(90,9 \%)$, possui formação generalista $(72,7 \%)$, obtiveram formação em instituição privada de ensino (100\%), possuem experiência em dispensação de medicamentos maior que 1 ano e menor que 5 anos (63,6\%), e a maio- 


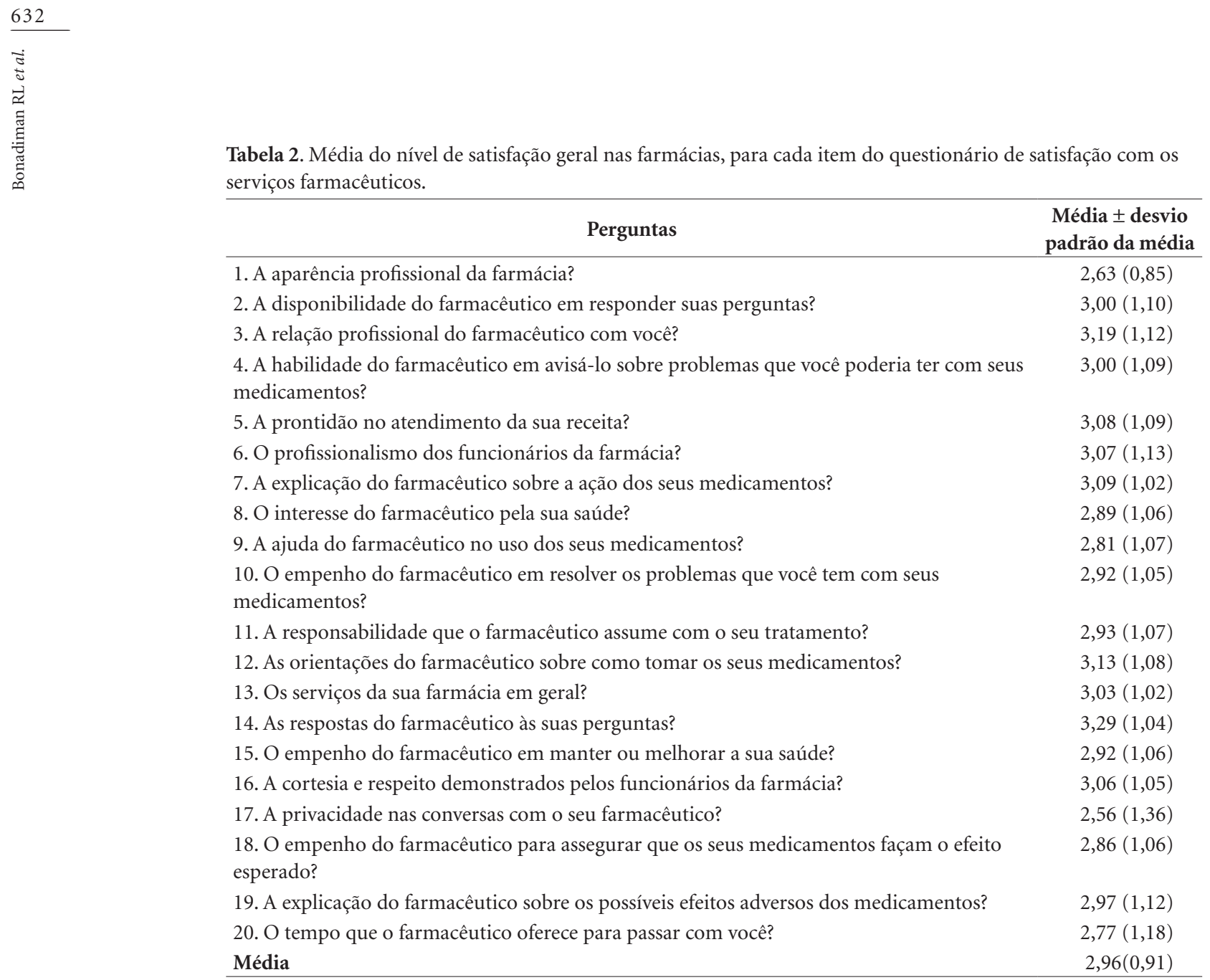

ria participou de congressos e cursos de curta duração $(81,9 \%)$ após o término da graduação.

Informações ocupacionais dos farmacêuticos também foram coletadas, onde 54,5\% da amostra trabalha de 30 a 40 horas/semana, ocupam o cargo a menos de 1 ano ou de 1 a 5 anos (45,5\%), a maioria $(63,6 \%)$ recebe salário entre $R \$ 840,00$ e R\$ 1.260,00 e não recebem benefícios, além de possuírem apenas um vínculo.

\section{Conhecimento dos farmacêuticos frente à dispensação}

A maioria dos farmacêuticos $(7 / 11=63,3 \%)$ acertou de 3 a 4 questões que norteavam sobre o conhecimento, sendo que nenhum farmacêutico errou todas as questões e apenas 1 farmacêutico $(9,1 \%)$ acertou as 5 questões propostas. Sobre os medicamentos sujeitos a controle especial, 18,2\% (2/11) acertaram e 81,8\% (9/11) erraram. Na dis- pensação de antimicrobianos, a maioria (7/11 63,6\%) obteve êxito e 4 farmacêuticos erraram.

Em relação à orientação do farmacêutico sobre a utilização dos medicamentos, 45,5\% acertaram e 54,5\% erraram. Sobre o descarte de medicamentos, todos os 11 participantes acertaram, entretanto quando questionados sobre o conceito de Atenção Farmacêutica, 18,2\% (2/11) deles não acertaram a resposta.

Foi possível verificar que 72,7\% (8/11) dos participantes afirmaram realizar a Atenção Farmacêutica nas farmácias básicas. Além disso, $54,5 \%$ (6/11) relataram que fazem registros contendo dados dos pacientes, orientações e intervenções realizadas. Em relação à estrutura física da farmácia, 63,6\% (7/11) dos farmacêuticos alegaram que isso dificulta o atendimento individualizado dos pacientes e que não há local adequado para realização desta prática.

O nível de conhecimento frente à dispensação de medicamentos foi classificado em: 36,4\% 
Tabela 3. Correlação das variáveis sociodemográficas, dados de atendimento e dispensação de medicamentos com a satisfação dos pacientes atendidos nas farmácias básicas do sul do Espírito Santo.

\begin{tabular}{|c|c|c|c|c|}
\hline Variáveis & Categorias & Insatisfeito & Satisfeito & $\mathbf{P}$ \\
\hline \multirow[t]{2}{*}{ Sexo } & Masculino & 75 & 17 & 0,444 \\
\hline & Feminino & 157 & 45 & \\
\hline \multirow[t]{6}{*}{ Escolaridade } & Analfabeto & 6 & 9 & 0,000 \\
\hline & Fundamental Incompleto & 38 & 25 & \\
\hline & Fundamental Completo & 57 & 6 & \\
\hline & Médio Incompleto & 33 & 5 & \\
\hline & Médio Completo & 85 & 15 & \\
\hline & Superior Completo & 13 & 2 & \\
\hline \multirow[t]{4}{*}{ Renda } & $<1$ salário & 41 & 19 & 0,000 \\
\hline & 1 a 2 salários & 161 & 38 & \\
\hline & 3 a 5 salários & 29 & 5 & \\
\hline & $>10$ salários & 1 & 0 & \\
\hline \multirow[t]{5}{*}{ Faixa etária } & 18 a 34 anos & 49 & 12 & 0,039 \\
\hline & 35 a 49 anos & 82 & 18 & \\
\hline & 50 a 60 anos & 71 & 16 & \\
\hline & 61 a 70 anos & 23 & 10 & \\
\hline & 71 a 80 anos & 7 & 6 & \\
\hline \multirow[t]{4}{*}{ Tempo de espera pelo atendimento } & 1 a 10 minutos & 114 & 60 & 0,000 \\
\hline & 11 a 20 minutos & 84 & 2 & \\
\hline & 21 a 30 minutos & 26 & 0 & \\
\hline & 31 a 40 minutos & 8 & 0 & \\
\hline \multirow[t]{3}{*}{ Tempo de duração do atendimento } & 1 a 5 minutos & 161 & 47 & 0,455 \\
\hline & 6 a 10 minutos & 25 & 9 & \\
\hline & 11 a 15 minutos & 2 & 0 & \\
\hline \multirow[t]{2}{*}{ Dispensação do medicamento } & Sim & 205 & 56 & 0,039 \\
\hline & Não & 24 & 6 & \\
\hline
\end{tabular}

A correlação foi considerada significativa quando $\mathrm{p}<0,05$.

$(\mathrm{n}=4)$ insatisfatório, $27,2 \%(\mathrm{n}=3)$ regular $\mathrm{e}$, $36,4 \%(\mathrm{n}=4)$, satisfatório.

\section{Condutas dos farmacêuticos}

As atividades não relacionadas diretamente à profissão farmacêutica, como limpeza e reposição de produtos nas prateleiras, são atividades realizadas com muita frequência, segundo informações fornecidas pelos participantes, e demandam muito tempo para serem concluídas (8/11 $=72,7 \%$ ), embora a atividade de verificação de validade, aquisição de produtos, orientação a paciente e a dispensação de medicamentos seja relatada como atividade realizada com muita frequência $(11 / 11=100 \%)$.

$\mathrm{Na}$ tabela 4 são apresentadas informações sobre as condutas dos farmacêuticos durante a dispensação de medicamentos. A maioria destes $(81,8 \%=9 / 11)$ relatou ter um relacionamento amigável com os funcionários e, 19,2\% (2/11) relatou ser difícil seguir as leis, devido à resistência imposta pelos funcionários do estabelecimento.

Quando questionados sobre como eles se avaliavam enquanto agentes de saúde comprometidos com o sucesso da farmacoterapia e qualidade de vida do paciente, $72,7 \%$, consideram que precisam melhorar quanto à orientação e o acompanhamento do tratamento medicamentoso.

\section{Discussão}

O principal resultado do presente estudo foi a determinação do baixo nível de satisfação dos usuários com relação aos serviços oferecidos pelas farmácias básicas, além disso, foi possível verificar uma relação entre o tempo de espera pelo atendimento e a satisfação do usuário. Adicionalmente, os farmacêuticos possuem conhecimento 


\begin{tabular}{|c|c|c|c|}
\hline \multirow[t]{14}{*}{ 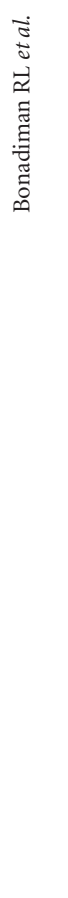 } & \multicolumn{3}{|l|}{ Tabela 4. Condutas relatadas pelos participantes durante a dispensação de medicamentos $(\mathrm{n}=11)$. } \\
\hline & Características & (n) & $(\mathbf{n}) \%$ \\
\hline & \multicolumn{3}{|l|}{ Conduta dos farmacêuticos durante a dispensação } \\
\hline & $\begin{array}{l}\text { - Analisa a prescrição, verifica informações como o uso de outros fármacos e a presença de } \\
\text { alergias antes da entrega do medicamento. Preocupa-se sempre em orientar sobre a forma } \\
\text { correta de uso. }\end{array}$ & 3 & 27,3 \\
\hline & $\begin{array}{l}\text { - Entrega o medicamento e anota na embalagem a forma correta de uso do produto. } \\
\text { Esclarecimentos adicionais apenas são fornecidos caso o paciente manifeste alguma dúvida. }\end{array}$ & 7 & 63,6 \\
\hline & - Apenas entrega o medicamento & 1 & 9.1 \\
\hline & \multicolumn{3}{|l|}{ Orientações fornecidas aos pacientes durante a dispensação } \\
\hline & - Posologia & 11 & 100 \\
\hline & - Interações medicamentosas & 4 & 36,4 \\
\hline & - Reações adversas & 7 & 63,6 \\
\hline & - Conservação do produto & 8 & 72,7 \\
\hline & \multicolumn{3}{|l|}{ Conduta quanto aos medicamentos próximos ao vencimento } \\
\hline & $\begin{array}{l}\text { - Recolher ou segregar em área específica. O paciente é alertado quando adquire um } \\
\text { produto nessas condições. }\end{array}$ & 5 & 45,5 \\
\hline & - Manter na prateleira juntamente com os medicamentos de prazo de validade superior. & 7 & 63,6 \\
\hline
\end{tabular}

entre satisfatório/regular para a dispensação de medicamentos, e acreditam que precisam melhorar quanto às ações de cuidados ao paciente.

Poucos são os estudos que verificam o nível de satisfação dos usuários em farmácias públicas de dispensação de medicamentos básicos e, de modo geral, estas pesquisas se concentram em farmácias comunitárias privadas e hospitalares $^{2,11,19}$. Entretanto, é importante contribuir com o campo de investigação da satisfação nestas farmácias públicas, e neste nível de atenção à saúde em países em desenvolvimento.

Em relação ao gênero, constatou-se que a maioria era do sexo feminino. A literatura descreve que as mulheres são maioria nos cuidados com a saúde e procuram mais por esses serviços $^{20-23}$. Além disso, Oliveira ${ }^{24}$ demonstrou que a predominância de mulheres na busca pelos serviços de saúde, pode ser associada à sua responsabilidade pelos cuidados de outros membros da família.

O baixo poder aquisitivo e instrucional se apresentou como característica principal, compreendendo indivíduos adultos entre os 35 e 60 anos. Fatores socioeconômicos são impactantes na qualidade de vida da população, pois têm influência direta no bem-estar do indivíduo, e ligado ao nível de satisfação acerca de um serviço recebido ${ }^{25}$.

De acordo com Mendonça et al. ${ }^{26}$, a satisfação dos pacientes com a assistência recebida pode ser influenciada pelas questões sociodemográficas, sendo observado maior nível de satisfação com maior renda familiar. No presente estudo, ao contrário, foi observado que menor renda e baixa escolaridade estão relacionadas a maiores níveis de satisfação. Isso pode ser explicado pelo fato de que, embora a constituição garanta a universalidade do direito à saúde, essa se mostra excludente, pois afasta pessoas com maior poder aquisitivo e informação, que estão insatisfeitas com a oferta e a qualidade dos serviços ${ }^{21}$.

A maior parte dos pacientes tiveram suas prescrições médicas com origem no SUS. Este resultado pode estar ligado ao tratamento de doenças crônicas como a hipertensão e diabetes ${ }^{27}$, e ainda, embora haja outras formas de assistência à saúde no Brasil, o SUS apresenta uma predominância absoluta no provimento desta assistência ${ }^{28}$.

No presente estudo, foi observado que quanto maior o tempo de espera, menor foi o nível de satisfação dos usuários, e que, a partir de 21 minutos, não houve mais usuários satisfeitos. Esses resultados se assemelham aos encontrados por Hassali et al. ${ }^{29}$ e Agu et al. ${ }^{30}$, onde o tempo de espera foi o principal fator que afetou o nível de satisfação dos pacientes.

Com relação à aquisição dos medicamentos, $89,7 \%$ dos pacientes conseguiram obtê-los no momento da dispensação e, 10,3\%, não conseguiram, devido à falta do medicamento. Diversos estudos demonstram o longo tempo de espera e também a falta de medicamentos para a dispensação em farmácias de unidades de saúde ${ }^{31-34}$. 
Para Ferri et al..$^{35}$, a demora para o atendimento é motivo de grande insatisfação por parte de quem usa os serviços de saúde. Esse fator deve ser levado em consideração, de modo que os planejamentos de ações nos serviços de saúde tenham como resultados a diminuição do tempo de espera, que pode aumentar o nível de satisfação dos pacientes ${ }^{29}$. De modo interessante, o tempo de duração do atendimento realizado pelo farmacêutico não apresentou relação com a satisfação dos usuários nos municípios.

Em farmácias básicas do sul do estado do Espírito Santo, os resultados demonstraram um baixo nível de satisfação, com a média geral de usuários insatisfeitos, contrastando com estudos de outros países, que indicam satisfação da maioria com os serviços de saúde recebidos, como os serviços de farmácia ${ }^{11,29,30,36}$, muito embora, no Brasil, 58\% da população se apresente insatisfeita com a assistência de saúde ${ }^{37}$.

Alturki e Khan ${ }^{2}$ demonstraram que avaliar a satisfação dos pacientes é um processo complexo, multifatorial, pois é muito difícil identificar um fator isolado diretamente associado com os níveis altos ou baixos de satisfação. Logo, vários são os motivos que podem ter contribuído com esse baixo nível de satisfação, como infraestrutura precária, falta de medicamentos, baixo número de farmacêuticos, e baixa qualidade nos serviços.

Malewski et al. ${ }^{11}$, observaram que farmacêuticos são procurados para aconselhamento, e o nível de satisfação dos pacientes pode estar diretamente ligado com suas expectativas e a percepção sobre como os serviços que são ofer$\operatorname{tados}^{38}$. Os pacientes precisam se sentir seguros nas habilidades dos farmacêuticos e isso pode ser alcançado com maior envolvimento destes nas atividades de promoção da saúde, que permitirá que os pacientes os enxerguem como indivíduos interessados por sua saúde e bem-estar ${ }^{11}$.

De acordo com o questionário, levando em consideração a média geral das farmácias, apesar de as maiores pontuações de satisfação terem sido relacionadas com aspectos do atendimento clínico do farmacêutico, as notas médias de cada questão não superaram o valor 3,3, que indica um baixo nível de satisfação.

As variáveis que tiveram as mais baixas avaliações da satisfação foram sobre a privacidade nas conversas e a aparência das farmácias. Berg e Donyai ${ }^{39}$ relataram que o relacionamento existente com o farmacêutico e o ambiente onde o atendimento é realizado, são elementos importantes que podem ter um papel chave na satisfação destes pacientes.
Nenhuma farmácia possuía local adequado para uma conversa privada com o farmacêutico, além de um ambiente completamente distante da humanização. Logo, estes motivos podem ter refletido o baixo nível de satisfação observado no presente estudo.

A percepção e a satisfação dos pacientes com os serviços farmacêuticos são fatores de extrema utilidade para avaliar os serviços oferecidos e, consequentemente, os resultados nos cuidados com a saúde ${ }^{30}$. Pacientes satisfeitos são mais propensos a terem melhor relação com o profissional de saúde, maior interesse sobre sua saúde e também a obterem melhores resultados ${ }^{40,41}$. Levando em consideração os resultados encontrados, é imperativa a adoção de medidas que promovam a melhoria dos serviços prestados e maior satisfação dos usuários.

Pesquisa realizada no município de Fortaleza-CE evidenciou que o principal problema ao se implantar o serviço de atenção farmacêutica no setor público é a ausência do profissional farmacêutico nos estabelecimentos de saúde ${ }^{27}$ e também que se exija o cumprimento da Lei 5991/73 ${ }^{42}$. Isto fica claro no presente estudo, quando em 8 municípios do Estado do Espirito Santo, apenas 11 profissionais farmacêuticos são responsáveis técnicos pelas farmácias básicas municipais, evidenciando a necessidade de contratação de mais profissionais.

Por vezes, as atividades burocráticas necessárias para o bom funcionamento da unidade acabam consumindo o tempo que deveria ser direcionado para o atendimento e acompanhamento dos pacientes, isto pode gerar um sentimento de frustração com a profissão, visto que fogem da realidade esperada por estes, além de prejudicar os serviços farmacêuticos ${ }^{43,44}$.

Lucchetta e Mastroianni ${ }^{8}$ observaram que a maioria dos farmacêuticos possuía nível de conhecimento regular. De acordo com a metodologia utilizada no presente estudo, os farmacêuticos tiveram conhecimento classificado como satisfatório/regular. Entretanto, a maioria errou em quesitos importantes, como orientação sobre a utilização dos medicamentos e controle especial, dado preocupante observado neste estudo.

Como os resultados demonstram que os farmacêuticos avaliados frequentaram especializações e tiveram alguma atualização, talvez o problema maior esteja na formação. Vale considerar o que afirma Silva e Vieira ${ }^{9}$, que a formação na área de atenção farmacêutica durante a graduação está deficiente, fazendo-se necessária a divulgação de informações sobre a legislação sanitária 
e que as faculdades de farmácia devem oferecer subsídios teóricos e práticos para que os futuros profissionais possam atuar na dispensação de medicamentos.

Ainda, para Lucchetta e Mastroianni ${ }^{8}$, faz-se necessário a readequação do projeto pedagógico dos cursos de farmácia, para que os alunos sejam formados com conhecimento e habilidade técnica, científica e legal, compatíveis com as diretrizes curriculares do curso.

Entretanto, na presente pesquisa, todos os farmacêuticos acertaram a questão referente à Atenção Farmacêutica. Um percentual de 54,5\% dos profissionais afirmou que realizam, e ainda $63,6 \%(7 / 11)$ alegaram que a estrutura física da farmácia dificulta o atendimento individualizado dos pacientes e que as mesmas não possuem local adequado para realização desta prática. Esses dados também foram reportados por Alano ${ }^{44}$, onde os serviços farmacêuticos foram prejudicados em razão da falta de espaço físico adequado e do pouco tempo disponibilizado aos pacientes, devido à realização de atividades burocráticas.

Quanto às condutas de dispensação, a maioria relatou dispensar e fornecer orientações caso o paciente apresente alguma dúvida, e que as orientações em sua maioria, dizem respeito à posologia e a reações adversas. Para Galato et al..$^{45}$, o conhecimento farmacêutico sobre o medicamento e as doenças são fatores determinantes para garantir uma dispensação de qualidade e que, o contrário, acarretaria na dispensação comprometida e de baixa qualidade.

Adicionalmente, essa referência de comportamento parece ser contraditória, de acordo com alguns pesquisadores que realizam a atenção farmacêutica. A prática profissional clínica é sistematizada e contínua, e não está de acordo com o comportamento de prestar assistência apenas diante de demanda do usuário e, também, não se restringe a prestação de informação sobre posologia e reações adversas ${ }^{46}$.

Apesar dos resultados encontrados, mais estudos sobre a satisfação de usuários de farmácias básicas, bem como de verificação do conhecimento dos farmacêuticos, se fazem necessários, tanto para avaliação da qualidade dos serviços prestados quanto para nortear melhorias que serão traduzidas em benefícios para saúde pública.

Sumariando, o nível de satisfação dos usuários de farmácias básicas é baixo, sendo o tempo de espera o fator diretamente ligado à satisfação do usuário. Além disso, o nível de conhecimento dos farmacêuticos frente à dispensação foi classificado satisfatório/regular, apesar de a maioria ter errado algumas questões importantes como a orientação sobre utilização dos medicamentos.

Em conclusão, o presente estudo apresentou evidências sobre o nível de satisfação dos pacientes, nas farmácias públicas municipais de dispensação de medicamentos básicos no interior do Espírito Santo, e como o tempo de espera do atendimento, não tem respondido às expectativas dos usuários. É importante destacar o conhecimento e a participação dos farmacêuticos na melhoria do sistema de saúde, principalmente na gestão de serviços culminando na melhoria da qualidade de dispensação de medicamentos.

\section{Limitações do estudo}

O número de profissionais farmacêuticos atuantes nas farmácias básicas apresentou-se muito reduzido, havendo ainda a não aceitação de alguns profissionais. Isso acarretou um número pequeno de profissionais para verificação do nível de conhecimento. Tal fato representou uma limitação importante para o estudo, pois impossibilitou análises de correlação estatística entre as avaliações relacionadas aos farmacêuticos e com o nível de satisfação dos usuários. Entretanto, tal fato (reduzido número de profissionais) e os resultados obtidos com a determinação do nível de conhecimento contribuíram para evidenciar a necessidade de mão-de-obra qualificada nos serviços de saúde. 


\section{Colaboradores}

RL Bonadiman, D Lenz, DC Endringer e TU Andrade trabalharam na concepção, projeto, análise e interpretação dos dados; elaboração do artigo ou revisão crítica de importante conteúdo intelectual e aprovação final da versão a ser publicada. AF Santanna trabalhou na análise e interpretação dos dados; elaboração do artigo ou revisão crítica de importante conteúdo intelectual e aprovação final da versão a ser publicada. EM Lima e GA Brasil trabalharam na elaboração do artigo, revisão crítica de importante conteúdo intelectual e aprovação final da versão a ser publicada.

\section{Agradecimentos}

Ao Professor Leonardo Regis Leira Pereira pela colaboração com o instrumento de verificação do nível de conhecimento dos farmacêuticos. Agradecemos à Fundação de Amparo à Pesquisa e Inovação do Espírito Santo (FAPES) pelo auxílio financeiro a esta pesquisa.

\section{Referências}

1. Reis EFB, Santos FP, Campos FE, Acúrcio FA, Leite MTT, Leite MLC, Cherchiglia ML, Santos MA. Avaliação da qualidade dos serviços de saúde: notas bibliográficas. Cad Saude Publica 1990; 6(1):50-61.

2. Alturki M, Khan TM. A study investigating the level of satisfaction with the health services provided by the Pharmacist at ENT hospital, Eastern Region Alahsah, Kingdom of Saudi Arabia. Saudi Pharm J 2013; 2(3):255-260.

3. Donabediam A. Evaluating the Quality of Medical Care. Milbank Q. 2005; 83(4):691-729.

4. Larson LN, Rover JP, MacKeigan LD. Patient satisfaction with pharmaceutical care: update of validated intrument. J Am Pharm Assoc 2002; 42(1):44-50.

5. Johnson JA, Coons SJ, Hays RD. The structure of satisfaction with pharmacy service. Med Care 1998; 36(2):244-250.

6. Correr CJ, Pontarolo R, Melchiors AC, Paula e Souza RA, Rossignoli P, Fernández-Llimós F. Satisfação dos usuários com serviços da farmácia: tradução e validação do "pharmacy services questionnaire" para o Brasil. Cad Saude Publica 2009; 25(1):87-96.

7. Reis TM, Guidoni CM, Girotto E, Rascado RR, Mastroianni PC, Cruciol JM, Pereira LRL. Knowledge and conduct of pharmacists for dispensing of drugs in community pharmacies: a cross-sectional study. Braz. J. Pharm. Sci 2015; 51(3):733-744.

8. Lucchetta RC, Mastroianni PC. Avaliação do conhecimento e das condutas dos farmacêuticos, responsáveis técnicos por drogarias. Rev Ciênc Farm Básica Apl 2010; 31(3):183-191.

9. Silva LR, Vieira EM. Conhecimento dos farmacêuticos sobre legislação sanitária e regulamentação da profissão. Rev Saude Publica 2004; 38(3):429-437.

10. Wal P, Wal A, Bhandari A, Pandey U, Rai AK. Pharmacist involvement in the patient care improves out come in hypertension patients. J Res Pharm Pract 2013; 2(3):123-129.

11. Malewski DF, Ream A, Gaither CA. Patient satisfaction with community pharmacy: comparing urban and suburban chain-pharmacy populations. Rev Social Adm Pharm 2014; 11(1):121-128.

12. Andrade TU, Burini DM, Mello MO, Bersácula NS, Saliba RAD, Bravim FT, Bissoli NS. Evaluation of the satisfaction level of patients attended by a Pharmaceutical Care Program in a Private Communitarian Pharmacy. Braz J Pharm Sci 2009; 45(2):349-355.

13. Brasil. Portal da Saúde [Internet]. Espírito Santo: Plano Diretor de Regionalização da Saúde. 2011. [acessado 2015 Mar 12]. Disponível em: www.saude.gov. br/download/PDR_PlanoDiretor de Regionalização _ES_2011.pdf

14. Brasil. Ministério da Saúde (MS). Política Nacional de Medicamentos. Brasília: MS; 2001.40 p.

15. Brasil. Ministério da Saúde (MS). Resolução no 338, de 6 de maio de 2004. Diário Oficial da União 2004; 7 maio.

16. Brasil. Ministério da Saúde (MS). Relação Nacional de Medicamentos Essenciais: Rename 2013. 8ª ed. Brasília: MS; 2013.

17. Kucukarslan S, Schommer JC. Patients' expectations and their satisfaction with pharmacy services. $J$ Am Pharm Assoc 2002; 42(3):489-495. 
18. Reis TM. Conhecimentos e condutas dos farmacêuticos para a dispensação de medicamentos e realização da Atenção Farmacêutica em drogarías [dissertação]. Porto Alegre: Universidade Federal do Rio Grande do Sul; 2013.

19. Naik-Panvelkar P, Saini B, Amour C. Measurement of patients satisfaction with community pharmacy services: a review. Pharm World Sci 2009; 31(5):525-537.

20. Pinheiro RS, Travassos C. Inequality in heatlh care use by the elderly in three districts in the city of Rio de Janeiro. Cad Saude Publica 1999; 15(3):487-496.

21. Vieira AC. Clientelismo e serviços de saúde. R. Pol. públ. 2002; 6(1):9-40.

22. Ibañez, N, Rocha JSY, Castro PC, Ribeiro MCSA, Forster AC, Novaes MHD, Viana ALA. Avaliação do desempenho da atenção no Estado de São Paulo. Cien Saude Colet 2006; 11(3):683-703.

23. Bonadiman RL, Bonadiman SL, Silva DA. Avaliação da adesão ao tratamento medicamentoso e não medicamentoso de pacientes hipertensos atendidos no PSF Guaritá, Itaperuna-RJ. Acta Biomedica Brasiliensia 2012; 3(1):73-84.

24. Oliveira EN. Saúde Mental e Mulheres: sobrevivência, sofrimento e dependência química lícita. $19^{\mathrm{a}}$ ed. Sobral: Edições UVA; 2000.

25. Joia LC, Ruiz T, Donalisio MR. Condições associadas ao grau de satisfação com a vida entre a população de idosos. Rev Saude Publica 2007; 41(1):131-138.

26. Mendonça KMPP, Guerra RO, Diógenes TPM. Influência das características sócio-demográficas na satisfação do paciente com o tratamento fisioterapêutico. Fisioter Mov 2006; 19(3):83-89.

27. Arrais PSD, Barreto LM, Coelho HLL. Aspectos de processos de prescrição e dispensação de medicamentos na percepção do paciente: estudo de base populacional em Fortaleza, Ceará, Brasil. Cad Saude Publica 2007; 23(4):927-937.

28. Correia LL. Atenção em saúde e eqüidade: uma análise de duas microrregiões assistenciais de saúde do Estado do Ceará. Rev Med Univ Fed Ceará 2000; 40:57-76

29. Hassali MA, Alrasheedy AA, Abrazak BA, Al-Tamimi SK, Saleem F, Ul haq N, Aljadhey H. Assesmentos general public satisfaction with public healthcare services in Kedah, Malaysia. Australas Med J 2014; 7(1):35-44.

30. Agu KA, Oqua D, Agada P, Ohiaeri SI, Adesina A, Abdulkareem MH, King RC, Wutoh AK. Assesment of pharmaceutical services in patients receiving antiretroviral therapy in outpatient HIV treatment setting. Int $J$ Clin Pharm 2014; 36(3):636-647.

31. Kloetzel K, Bertoni AM, Irazoqui MC, Campos VPG, Santos RN. Controle de qualidade em atenção primária à saúde. I - A satisfação do usuário. Cad Saude Publica 1998; 14(3):623-628.

32. Instituto Brasileiro de Opinião Pública e Estatística (IBOPE). Atendimento nas unidades públicas de saúde. Seção Opinião Pública - Área: Análises e indices/2000. [acessado 2014 Jul 21]. Disponível em: http://www. ibope.com.br/calandraWeb/servlet/CalandraRedirect? temp $=6 \&$ proj $=$ PortalI

33. Cerdá JMV, Gimeno GS, Boquet EM, Villalba EMF, Alminãna MA. Satisfacción percebida por pacientes infectados por el VUH conlaunidad de atención farmacêutica a pacientes externos (UFPE). Farm Hosp 2005; 29(2):134-139.
34. Bonadiman RL, Bonadiman RL, Bonadiman SL, Silva DA. Estudo das prescrições medicamentosas em uma farmácia básica de Itapemirim, Espírito Santo - Brasil. Acta Biomedica Brasiliensia 2013; 4(2):114-123.

35. Ferri SMN, Pereira MJB, Mishima SM, Caccia-Bava MCG, Almeida MCP. As tecnologias leves como geradoras de satisfação em usuários de uma unidade de saúde da família. Interface (Botucatu) 2007; 11(23):515529.

36. Mcfarland MS, Wallace JP, Parra J, Baker J. Evaluation of patient satisfaction with Diabetes management provided by clinical pharmacist in the patient-centered medical home. Patient 2014; 7(1):115-121.

37. Szwarcwald CL, Viacava F, Vasconcellos MTL, Leal MC, Azevedo LO, Queiroz RSB, Carvalho MF, Medeiros MGPF, Frias PG, Gama SGN, Souza Junior PRB, Lansky S, Luhm KR, Mazzei MC, Ribeiro MCSA, Santos S, Souza WV, Theme Filha MM. Pesquisa Mundial de Saúde - 2003: O Brasil em números. RADIS 2004; 1(23):14-33.

38. Ikegami N, Kawakita H. Patient satisfaction and hospital management policy. Jpn Hosp 1987; 6:11-15.

39. Berg MVD, Donyai PA. A conceptual framework of patient satisfaction with a pharmacy adherence service. Int J Clin Pharm 2014; 36(1):182-191.

40. Horvat N, Kos M. Development and initial validation of a patient satisfaction with pharmacy performance questionnaire (PSPP-Q). Eval Health Prof 2010; 33(2):197-215.

41. Horvat N, Kos M. Slovenian pharmacy performance: a patient-centred approach to patient satisfaction survey content development. Int J Clin Pharm 2011; 33(6):985-996.

42. Brasil. Lei n. 5991, de 17 de dezembro de 1973. Dispõe sobre o controle sanitário do comércio de drogas, medicamentos, insumos farmacêuticos e correlatos, e da outras providências. Diário Oficial da União 1973; $19 \mathrm{dez}$.

43. Oliveira AB, Oyakawa CN, Miguel MD, Zanin SMW, Montruchio DP. Obstáculos da atenção farmacêutica no Brasil. Rev Bras Cienc Farm 2005; 41(4):409-413.

44. Alano GM. Reflexão e contribuição para uma nova prática: os serviços farmacêuticos voltados ao paciente sob a perspectiva de farmacêuticos do Estado de Santa Catarina [dissertação]. Florianópolis: Universidade Federal de Santa Catarina; 2005.

45. Galato D, Alano GM, Trauthman SC, Vieira AC. A dispensação de medicamentos: reflexão sobre o processo para prevenção, identificação e resolução de problemas relacionados à farmacoterapia. Rev. Brasil. Cienc. Farm. 2008; 44(3):465-475.

46. Dáder MJF, Hernández DS, Castro MMS. Método Dáder. Guía de seguimiento farmacoterapéutico. Grupo de Investigación en Atención Farmacéutica. $3^{\text {a }}$ ed. Granada: Universidad de Granada; 2007.

Artigo apresentado em 04/09/2015

Aprovado em 18/05/2016

Versão final apresentada em 20/05/2016 